\title{
Model of contact friction based on extreme value statistics
}

\author{
A. MALEKAN ${ }^{*}$, S. ROUHANI \\ Department of Physics, Sharif University of Technology, Tehran 11165-9161, Iran \\ Received: 29 November 2017 / Revised: 28 January 2018 / Accepted: 10 March 2018 \\ (C) The author(s) 2018. This article is published with open access at Springerlink.com
}

\begin{abstract}
We propose a model based on extreme value statistics (EVS) and combine it with different models for single-asperity contact, including adhesive and elasto-plastic contacts, to derive a relation between the applied load and the friction force on a rough interface. We determine that, when the summit distribution is Gumbel and the contact model is Hertzian, we obtain the closest conformity with Amonton's law. The range over which Gumbel distribution mimics Amonton's law is wider than that of the Greenwood-Williamson (GW) model. However, exact conformity with Amonton's law is not observed for any of the well-known EVS distributions. Plastic deformations in the contact area reduce the relative change in pressure slightly with Gumbel distribution. Interestingly, when elasto-plastic contact is assumed for the asperities, together with Gumbel distribution for summits, the best conformity with Amonton's law is achieved. Other extreme value statistics are also studied, and the results are presented. We combine Gumbel distribution with the GW-McCool model, which is an improved version of the GW model, and the new model considers a bandwidth for wavelengths $\alpha$. Comparisons of this model with the original GW-McCool model and other simplified versions of the Bush-Gibson-Thomas theory reveal that Gumbel distribution has a better conformity with Amonton's law for all values of $\alpha$. When the adhesive contact model is used, the main observation is that there is some friction for zero or even negative applied load. Asperities with a height even less than the separation between the two surfaces are in contact. For a small value of the adhesion parameter, a better conformity with Amonton's law is observed. The relative pressure increases for stronger adhesion, which indicates that adhesion-controlled friction is dominated by load-controlled friction. We also observe that adhesion increases on a surface with a lower value of roughness.
\end{abstract}

Keywords: Amonton's law; contact mechanics; extreme value statistics; friction

\section{Introduction}

Friction between solid bodies is an extremely complex physical phenomenon, acting on many scales [1-5]. Amonton claimed that frictional force is proportional to the normal load and is independent of the apparent contact surface, relative velocity, and temperature. In other words, there is a linear dependence between normal load and friction force for a wide range of loads and friction coefficient is merely dependent on the material of the two surfaces in contact [6]. Various settings [7-9] were used to test these claims. Amonton's law does not hold completely true in all cases. However, for the first order of approximation, the friction law is formulated very simply as

$$
f=\mu F
$$

where $\mu$ is the friction coefficient and $F$ is the normal load. This first-order approximation serves many engineering applications. However, its physical basis remains a mystery. It is known that many qualifications to this simple relation hold. Coulomb discovered that the static frictional force between two surfaces increases with the contact time $[10,11]$. The creep process is a possible mechanism that leads to this phenomenon. Owing to creep processes, the real contact area grows with time and this growth is faster at higher temperatures [12]. Hence, the static frictional force has a

* Corresponding author: A. MALEKAN, E-mail: malekan_azadeh@physics.sharif.edu 


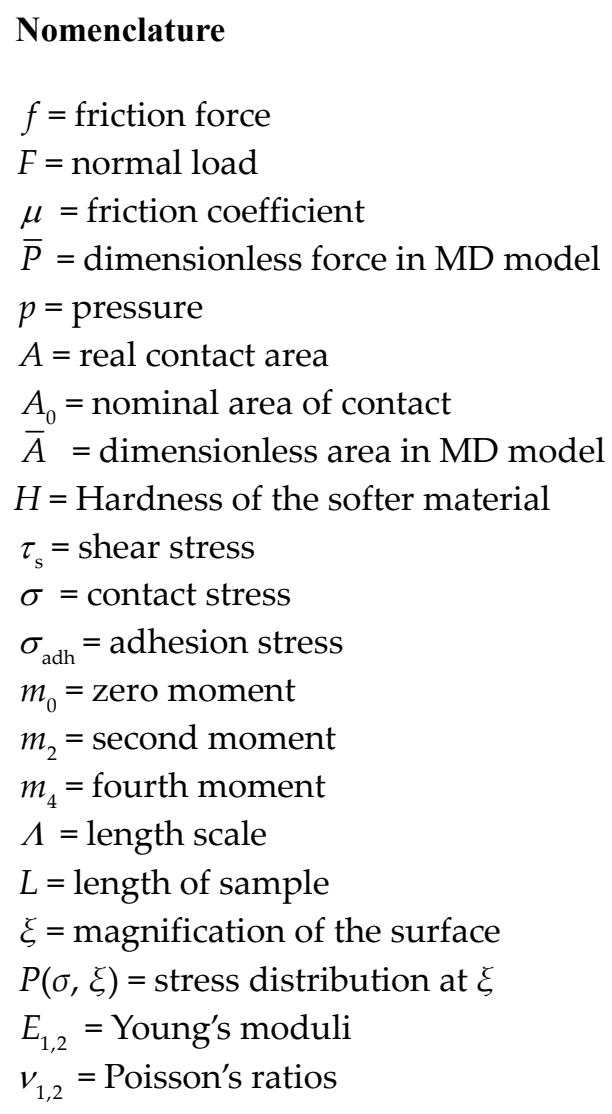

$d=$ sepration between two surfaces

$E^{*}=$ effective Young's moduli

$N_{0}=$ total number of asperities

$\alpha=$ bandwith of wavelengths

$R_{\mathrm{G}}=$ Greenwood model's radius

$R_{\mathrm{A}}=$ NT's model radius

$R_{1,2}=$ asperity raduis

$\omega=$ surface roughness

$\alpha_{k}=$ the zeros of Airy function

$U(a, b, c)=$ confluent hyper geometric function

$d_{\max }=$ the highest summit limit

$\eta=$ density of asperities

$t=$ dimensionless distannce $\frac{d}{\sqrt{m_{0}}}$

$\delta=$ interference of two surfaces

$\delta_{\mathrm{c}}=$ critical interference

$\psi=$ plasticity index

$\Delta \gamma=$ surface energy

$z_{0}=$ the equilibrium in Lennard - Jones force

$\lambda=$ adhesion parameter logarithmic dependence on time, because an increase in the contact area reduces the speed of the creep process [13]. The linear dependence of the basic frictional force is not valid for all force domains. Although the linearity holds for several orders of magnitude of the normal load for metallic materials [14], it breaks down for materials such as polymers and elastomers or soft metals [15]. The frictional force is not independent of roughness. It shows a negligible dependence on it. The friction coefficient for extremely smooth metal surfaces is larger than that for rough surfaces [8]. In addition, further deviations from the simple Amonton's law have been observed in rubber, which exhibits unusual asymmetry in the friction direction [9]. With regard to sliding friction, in the first approximation, the coefficient of friction is independent of speed [6], although experiments show that friction force has some dependency on the sliding velocity. Friction force remains constant for moderate velocities whereas it decreases for high velocities. For very small velocities, an increase in velocity results in an increase in friction force [6]. Various dynamic models were suggested to explain the velocity dependence of friction $[16,17]$.

In addition to all the aforementioned deviations from Amonton's law, extensive theoretical efforts have been made to substantiate Amonton's claim [17, 18]. One of the early explanations of Amonton's law was given by Bowden and Tabor [14]. Actual contact occurs only at the summits because of surface roughness. They considered complete plastic contact and therefore, the actual area of contact is connected to hardness indentations. The total area of actual contact $A$ is $A=F / H$, where $H$ is the hardness of the softer material and $F$ is the normal load. The frictional force is $f=\tau_{\mathrm{s}} F / H$, and the local shear stress is $\tau_{\mathrm{s}}$. They proposed a coefficient of friction $\mu=\tau_{\mathrm{s}} / H$, as the ratio of two material properties.

As real surfaces are rough on the microscopic scale, contact occurs at the summits of asperities. The GW model proposed an elastic and adhesion-less asperity contact with Gaussian distribution for the heights of summits. The authors Greenwood and Williamson observed an approximately constant pressure during 
loading [19]. Archard simulated a rough surface as a series of spheres superimposed hierarchically [20]. He proved that the relation between the real contact area $A$ and the normal load $F$ is given by a power law, $A \sim F^{\alpha}$, where the exponent $\alpha \approx 1$ in the case of a complex real surface $A$ is nearly proportional to the load, according to Amonton's law. Bush, Gibson, and Thomas (BGT) [21] used a statistical theory of isotropic randomly rough surfaces that utilizes a bandwidth parameter. They used Longuet-Higgins [22] and Nayak [23] probability distribution of summits for the surface statistics of an isotropic surface.

$$
\begin{aligned}
P\left(z, R_{1}, R_{2}\right)= & \frac{\sqrt{27}}{(4 \pi)^{2}} \frac{1}{m_{2} m_{4} \sqrt{m_{0} m_{4}}} C_{1}^{1 / 2} \exp \left[-C_{1}\left(\frac{z}{m_{0}^{1 / 2}}\right.\right. \\
& \left.\left.+\frac{3}{2 \sqrt{\alpha}} \frac{1 / R_{1}+1 / R_{2}}{m_{4}^{1 / 2}}\right)^{2}\right]\left|\left(\frac{1}{R}\right)_{1}-\left(\frac{1}{R}\right)_{2}\right| \\
& \times\left(R_{1} R_{2}\right)^{-1} \exp \left\{-\frac{3}{16 m_{4}}\left[3\left(1 / R_{1}+1 / R_{2}\right)^{2}\right.\right. \\
& \left.\left.-8\left(R_{1} R_{2}\right)^{-1}\right]\right\}
\end{aligned}
$$

Power spectral density (p.s.d.) is the Fourier transform of height autocorrelation function for a Gaussian and isotropic surface, $z$ is the summit height, and $R_{1}, R_{2}$ are summit radii. The zero, second, and fourth moments $-m_{0}, m_{2}$, and $m_{4}$, respectively - of the surface roughness power spectrum are functions of the breadth of the surface roughness and wavelength $\alpha=\frac{m_{0} m_{4}}{m_{2}^{2}}$.

Longuet-Higgins has shown in a random and isotropic surface that $\alpha \geq 3 / 2$. The p.s.d. spreads with the increase in $\alpha$. In BGT theory, an isotropic rough surface with joint summit and curvature distribution has been assumed by Longuet-Higgins and Nayak [23]. This surface is considered to be in contact with a flat surface. The spheres of the GW model are replaced by paraboloids. The contact area $A$ is proportional to the normal load [21], provided that the normal applied load is very low or $A$ is well below the apparent area of contact.

Persson [24] linked the apparent contact area $A$ to a length scale $\Lambda$. The length $\Lambda$ is the projection of the contact area when the original surface considered is smooth on all length scales below $\Lambda$. The ratio $\xi=L / \Lambda$ is the magnification of the surface, where $L$ is the length of the sample. Persson assumed that $P(\sigma, \xi)$, the stress distribution at the magnification $\xi$, satisfies a diffusion-like equation. He also observed a linear relationship between the normal load and the real area of contact, provided that the normal applied load is small.

In this paper, we propose a model for friction based on extreme value statistics (EVS) [25]. The rough contact friction force is given by two considerations: the model for asperity contact and the summit distribution. The simplest choice for single-asperity contact is an elastic contact model or Hertzian asperity [26]. The others are adhesive and elastic-plastic contacts models; the Maugis-Dugdale (MD) [27] model is a general adhesive theory and Johnson-Kendall-Roberts (JKR) and Derjaguin-Muller-Toporov (DMT) are its limiting cases. Another option is the Chang-EtsionBogy (CEB) [28] or elastic-plastic model based on volume conservation of an asperity during plastic deformation. We use EVS for independent and identically distributed (IID) variables and the maximum height $h_{\mathrm{m}}(1+1)$ Kardar-Parisi-Zhang (KPZ) model. We follow the GW model assumptions (see below) and combine the various possibilities of asperity contact and EVS distributions and solve numerically to obtain a relationship between the contact area, friction force, and applied load for various distributions and contacts. As there is no direct evidence for which EVS distribution is to be used, we test various universal EVS distributions to determine the one that produces better conformity with Amonton's law. In addition, we use EVS for summit distribution in some simplified version of BGT models, which consider a wavelength for the radius of the summit. The Gumbel distribution with an elasto-plastic contact is considered to be the most suitable distribution. Notably, surface correlations are ignored in this kind of analysis, with the exception of the KPZ surface.

This paper is organized as follows. In Section 2, we describe the Greenwood-Williamson (GW) Model, which sets the basis for our analysis. In Section 3, we provide a brief introduction to EVS. In Section 4, we combine EVS with single-asperity models and numerically calculate the contact pressure for numerous universal EVS and different asperity models. In Section 5, we attempt to question the assumptions of 
the GW model. We conclude this paper with some concluding remarks.

\section{Greenwood-Williamson model}

Greenwood and Williamson [19] developed a theory based on Hertz contact theory, assuming a flat rigid plane in contact with a rough surface where the distance between the flat rigid planes from the mean height of the rough surface is $d$. All asperities have the same radius $R$. The height of the peaks is stochastically distributed around an average value (Fig. 1). If $\Phi(z)$ is the summit distribution and there are $N_{0}=\eta A_{0}$ asperities within a nominal area of $A_{0}$, the total real area of contact is

$$
A=\pi N_{0} \int_{d}^{\infty} \mathrm{d} z \Phi^{*}(z) R(z-d)
$$

Further, the total load is the summation of loads of every single-asperity in contact.

$$
F=\frac{4}{3} N_{0} E^{*} \int_{d}^{\infty} \mathrm{d} z \Phi^{*}(z) \sqrt{R}(z-d)^{3 / 2}
$$

where $E^{*}$ and $R$ are defined as

$$
\begin{gathered}
\frac{1}{E^{*}}=\frac{1-v_{1}^{2}}{E_{1}}+\frac{1-v_{2}^{2}}{E_{2}} \\
\frac{1}{R}=\frac{1}{R_{1}}+\frac{1}{R_{2}}
\end{gathered}
$$

Assume a Gaussian distribution for the summits of asperities [19].

$$
\Phi^{*}(z)=\left(\frac{1}{2 \pi \omega^{2}}\right)^{\frac{1}{2}} \exp \left(-\frac{z^{2}}{2 \omega^{2}}\right)
$$

It is better to use the natural length scale of the problem, namely the roughness $\omega$ (RMS of the height of asperities), as a dimensional quantity. Moreover,

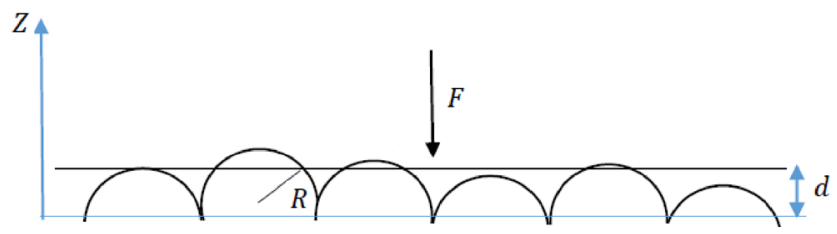

Fig. 1 The schematic drawing of GW model. the real area of contact becomes

$$
A=\pi \omega N_{0} R \int_{\frac{d}{\omega}}^{\infty} \mathrm{d} z \Phi(z)\left(\frac{z}{\omega}-\frac{d}{\omega}\right)
$$

The total load after scaling with the roughness obtained is

$$
F=\frac{4}{3} N_{0} E^{*} \omega^{3 / 2} R^{1 / 2} \int_{\frac{d}{\omega}}^{\infty} \mathrm{d} z \Phi(z)\left(\frac{z}{\omega}-\frac{d}{\omega}\right)^{3 / 2}
$$

Further, $\Phi(z)=\omega \Phi^{*}(\omega z)$. The load divided by the actual area of contact $\frac{F}{A}$ versus the surface separation is plotted where $\varphi(z)$ is a Gaussian distribution (Fig. 2). If we assume that the actual area of contact is proportional to the friction force, this plot should provide us the friction coefficient.

\section{Extreme value statistics}

The assumption that the summit distribution is Gaussian is too simple in the GW model. Given a height distribution, we must consider distribution in summits as the asperities are in contact at their summits. This distribution is described by EVS.

EVS is a branch of statistics that strives to determine the probability distribution of maxima and minima of given distributions. Given a random height distribution, we intend to determine the distribution of its maxima. This is given by the EVS of $\varphi(x)$. EVS has many applications in natural phenomena and engineering

$$
\frac{F}{A}\left(\frac{3 \pi}{4 E^{*}}\right)\left(\frac{R}{\omega}\right)^{1 / 2} \quad \text { Gaussian distribution }
$$

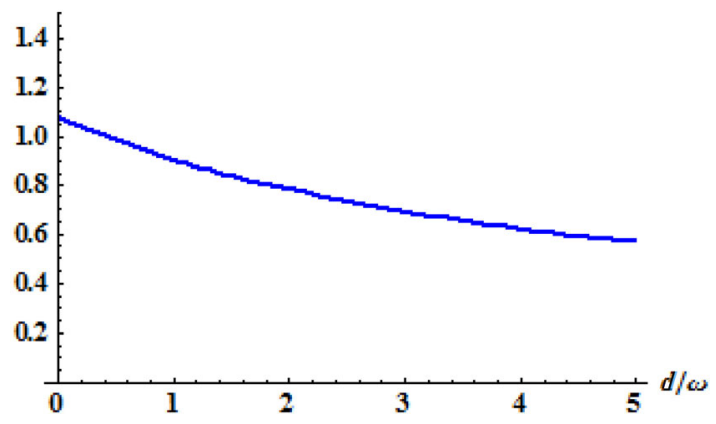

Fig. 2 The pressure of contact as separation of surfaces when the Gaussian distribution is summits' distribution. Clearly, the friction coefficient is not independent of the load, though there is the almost constant behavior for the range of $(2.5 \omega-5 \omega)$. 
[29-32], and it might be an appropriate choice for the summit distribution. Unfortunately, EVS of any given height distribution might not be known, but it is known for some special cases. We will investigate these special cases and estimate for the general cases. Based on the mother distribution, there are three types of universal limit distributions for IID and for numerous random variables. This is known as Gnedenko's classical law of extremes [33]. The probability density function (PDF) of the maxima is given by Fisher-Tippett-Gumbel distribution [34], when the distribution of IID variables has tails decaying faster than power law but are unbounded such as $P(x) \sim e^{-x \delta}$ with $\delta>0$.

$$
f_{1}(z)=e^{-\frac{x}{\omega}-e^{-\frac{x}{\omega}}}, \quad \omega>0, \quad x \in(-\infty, \infty)
$$

The Gumbel universality class corresponds to exponential, Gaussian, or gamma distribution of variables. It describes extreme wind speeds, sea wave heights, floods, rainfall, etc. In addition, it has applications in size phenomena, such as the size of material flaws and surface imperfections, and event magnitudes, such as queue length and order lead time [29].

For IID random variables, with the parent distribution of power law convergence $P(x) \sim x^{-(1+\beta)}$ with $\beta>0$, the PDF is Fréchet distribution given by

$$
f_{2}(x)=\frac{\beta}{\omega}\left(\frac{x}{\omega}\right)^{-\beta-1} e^{-\frac{x^{-\beta}}{\omega}}, \quad \omega>0, \quad x \in[0, \infty)
$$

The Fréchet domain has distributions with an infinite, yet heavier tail than the exponential distributions. This corresponds to EVS of Cauchy or Preto distributions. The Fréchet distribution can be applied to extreme events such as annually maximum one-day rainfalls and river discharges [33]. The maximum loads that can be tolerated by engineering devices are required in their service mission [30]. Natural phenomena such as floods, snow accumulation, wave forces, earthquakes, and wind pressure often cause these loads [29]. The intrinsic longer upper tail of Fréchet distribution leads to an upward data fit.

Therefore, the Fréchet distribution is another alternative for modeling maximum extreme value phenomena in addition to the Gumbel distribution.

For the parent distributions with bounded tails such as $P(x) \stackrel{x \rightarrow a}{\rightarrow}(a-x)^{\beta-1}$ with $\beta>0$, the PDF is the Weibull distribution.

$$
f_{3}(x)=\beta\left(\frac{x}{\omega}\right)^{(\beta-1)} e^{-\left(\frac{x}{\omega}\right)^{\beta}}, \quad \omega>0, \quad x \in[0, \infty)
$$

Distributions in this universality class have lighter tails than exponential distribution, which has a finite upper bound. There are several papers about the applications of Weibull distribution in natural phenomena such as wind-speed data analysis [31], earthquake magnitude analysis [32], and volcanic occurrence data.

However, many distributions do not belong to the three aforementioned domains of attraction. For example, EVS of geometric and Poisson distributions cannot be determined by the standard extreme value distributions. EVS domains of attraction include most applied distributions, such as Pareto-like distributions (Cauchy), normal, and Beta distributions [35].

A general theory similar to that for IID does not exist for strongly correlated random variables. There are a few examples, such as maximum heights of a fluctuating $(1+1)$ dimensional interface, where the EVS of a strongly correlated system was computed exactly. Majumdar $[36,37]$ determined that the PDF of maximum height $h_{\mathrm{m}}(1+1) \mathrm{KPZ}$ model has the scaling form for all $L \omega$.

$$
P\left(h_{m}, L \omega\right)=\frac{1}{\sqrt{L \omega}} f\left(\frac{h_{m}}{\sqrt{L \omega}}\right)
$$

The scaling function named as Airy distribution indicates

$$
f(x)=\frac{2 \sqrt{6}}{x^{\frac{10}{3}}} \sum_{k=1}^{\infty} e^{\frac{-b_{k}}{x^{2}}} b_{k}^{\frac{2}{3}} U\left(\frac{-5}{6}, \frac{4}{3}, \frac{b_{k}}{x^{2}}\right)
$$

where $\omega$ is the surface roughness, $L$ is the length of the sample, $b_{k}=\frac{2}{27} \alpha_{k}^{3}$, where $\alpha_{k}$ represents the absolute values of the zeros of the Airy function, and $U(a, b, z)$ is a confluent hyper geometric function of the second kind.

\section{Extreme value statistics model of friction}

Let us now repeat the GW model with EVS distributions as $\Phi(z)$. Furthermore, we will consider various 
asperity contacts and combine them with EVS. As the distribution of the heights is not known, the relevant EVS distribution is also not known. Therefore, we shall report the results of the three universal EVS distributions here.

At a separation $d>d_{\max }$, the two surfaces are no longer in contact; hence, the normal load vanishes. As all EVS distributions except Fréchet fall quickly, the integral of the load and contact area converges, and $\frac{d_{\max }}{\omega}$ is replaced by infinity. We plot the dimensionless pressure $\frac{4}{3}\left(E^{*} / \pi\right)^{*}(\omega / R)^{1 / 2} *(F(d, R) / A(d, R))$ as a function of $d / \omega$. A decrease in the normal load and the real area of contact with the increase in $d$ suggests that the ratio may be approximately constant. However, Amonton's law contradicts this suggestion. The linear relationship between the real area of contact and applied load is expected in this interval. Fréchet distributions with $0 \leq \beta \leq 2$ have a fat tail; thus, to determine the total load and real area of contact, we must set an upper limit to the peak height $d_{\max }$. It is assumed that the bigger the area of the sample, the likelier it is to encounter a higher maximum peak. The scaling of this maximum with sample size is related to the falls of the distribution at large values. Here, we assume that it scales with the nominal area, $d_{\max } \sim A_{0}^{\gamma}$. Fréchet distribution scales with size as $N_{0}^{\frac{1}{\beta}}$; thus, $N_{0}$ is proportional to $A_{0}$. We should introduce a cut off for the maximum height, and hence, $d_{\max }$ is chosen such that $99 \%$ of summits included $d_{\max } \sim A_{0}^{\frac{1}{\beta}}$.

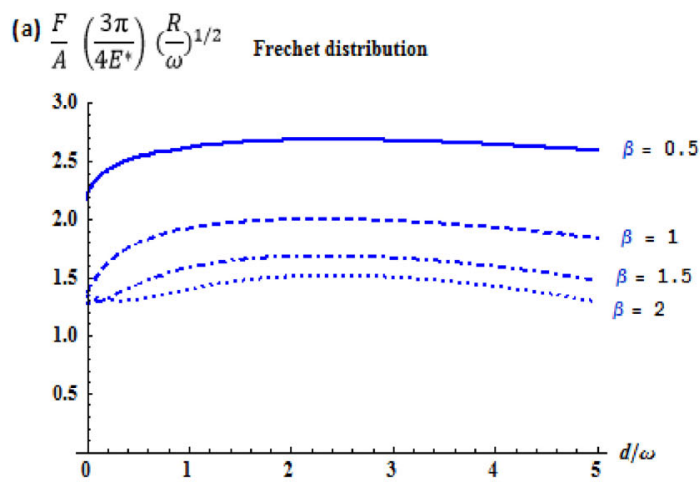

We plot the nominal friction force as a function of separation for the Fréchet distribution. The resulting friction force does not depend on $d_{\max }$ or equivalently on the nominal area of contact. The friction does not show a monotonous trend in Fig. 3(a). In Fréchet distribution with $\beta>2$, with the increase in load, the number of short summits is not sufficient to reduce or balance the pressure in high loads (Fig. 3(b)). Figure 4 shows the pressure of contact when the other EVS is used as the summit distribution. Gumbel distribution has the most uniform pressure in the physical contact condition and shows the best conformity with Amonton's law (Fig. 4(a)). The Gumbel domain of attraction belongs to mother distributions with an exponential decay such as Gaussian. This result is consistent with the observations that suggest that Gaussian distribution is the height distribution of asperities.

For $\beta=1$ the Weibull distribution is the exponential distribution, which-independent of the particular surface model-shows the exact proportionality between the load and the area of contact (Fig. 4(b)), although it is not a fair approximation of the asperities of the surfaces as this indicates uniform distribution of the height of asperities [19].

Simplified EVS distribution for 1+1 KPZ model (Airy distribution, not to be confused with airy function) for a surface is $[36,37]$

$$
\varphi(z)= \begin{cases}0.15^{*}\left|a_{1}\right|^{2} z^{\frac{-10}{3}} e^{\frac{-2 a_{1}^{3}}{27 z^{2}}} & z \leq 0.56 \\ 1.84 e^{-6 z^{2}} & z>0.56\end{cases}
$$

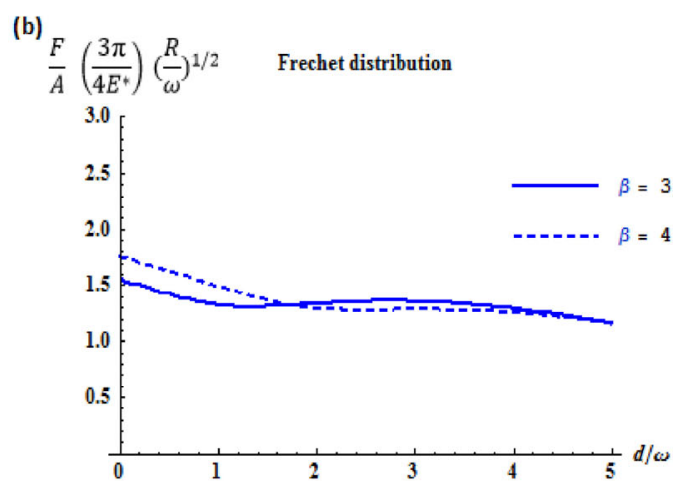

Fig. 3 The pressure of contact for the Fréchet distribution and Hertzian contact. (a) The pressure of contact does not have a monotonous trend by increasing load for $\beta<2$. (b) For $\beta \geq 2$, the pressure of contact has a rise in pressure due to a reduction in the number of short summits for larger separations. 
(a) $\frac{F}{A}\left(\frac{3 \pi}{4 E^{*}}\right)\left(\frac{R}{\omega}\right)^{1 / 2}$ Gumbel distribution

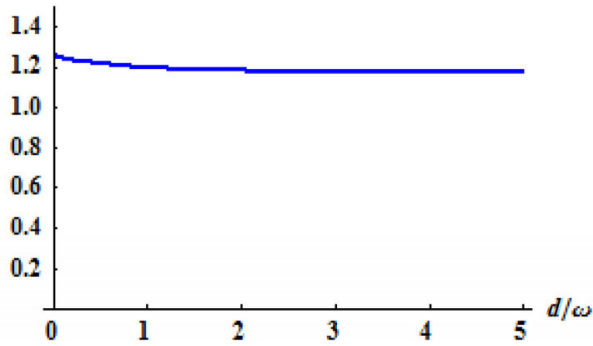

(b) $\frac{F}{A}\left(\frac{3 \pi}{4 E^{*}}\right)\left(\frac{R}{\omega}\right)^{1 / 2} \quad$ Weibull distribution

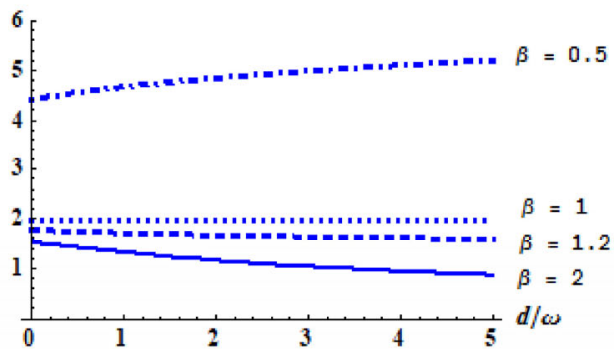

Fig. 4 Different Weibull and Gumbel distributions as summits distribution with Hertzian contact. (a) Gumbel distribution gives an almost flat pressure which indicates the validity of Amontons' law for the range of $(\omega, 5 \omega)$. (b) The Weibull distributions have different trends for various values of beta. $\beta=1$ corresponds to the exponential distribution which shows exact proportionality between the load and the area of contact independent of the surface model. There is also good correspondence with Amontons Law values of beta near one (Fig. 6(b)).

where $a_{1}$ is the first zero of the Airy function. In Fig. 5, Airy distribution is the summit distribution with Hertzian contact. Airy distribution is not a good candidate for summit distribution as the pressure changes are larger than those in other EVS and even Gaussian distribution. In Table 1, we can observe the range of total load and real area of contact when Gumbel distribution is used as the summit distribution for two surfaces with $E_{1}=E_{2}=17 \mathrm{GPa}$ and $v_{1}=v_{2}=$ 0.15 . It can be observed that relative pressure change in the interval of $(\omega, 5 \omega)$ is 0.02 for Gumbel distribution. These values are consistent with the experimental observation by Nuri and Hailing [38]. We observe that the typical loads are reasonable when our model is in good conformity with Amonton's law. Therefore, Gumbel distribution is best suited and fits Amonton's law. However, elastic contact conserves energy and cannot be a good candidate for friction; thus, a plastic component to the asperity behavior is necessary.

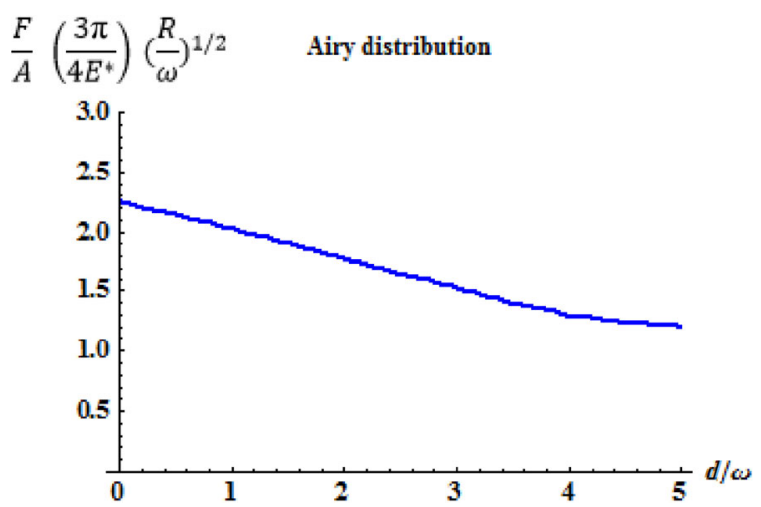

Fig. 5 Airy distribution has used as summits' distribution with Hertzian contact. In comparison with other EVS distributions and even Gaussian distribution, it has the biggest variation in pressure.
Table 1 The total load and real area of contact for concrete with Gumbel summits' distribution in $\omega$ and $5 \omega$.

\begin{tabular}{cccc}
\hline$\eta R \omega$ & $\frac{\omega}{R}$ & $\begin{array}{c}F(5 \omega)-F(\omega) \\
(\mathrm{kg})\end{array}$ & $\frac{A_{\mathrm{re}}\left(d_{2}\right)}{A_{0}}-\frac{A_{\mathrm{re}}\left(d_{1}\right)}{A_{0}}$ \\
\hline 0.0302 & $8.75 \times 10^{-5}$ & $0.0005-80$ & $0.02-0.0001$ \\
0.0374 & $2.00 \times 10^{-4}$ & $0.9-150$ & $0.02-0.0001$ \\
0.0601 & $1.77 \times 10^{-3}$ & $4.5-710$ & $0.03-0.0002$ \\
0.0401 & $2.48 \times 10^{-4}$ & $1.0-177$ & $0.02-0.0001$ \\
\hline
\end{tabular}

Here, we use the CEB model [28] of elastic-plastic contact based on volume conservation of plastically deformed region of the asperity. Figure 6(a) compares the pressure of the elastic-plastic model with that of the Hertzian model. As the plastic index increases, the pressure decreases. Increasing the real area of contact owing to plastic deformation makes the pressure more uniform. Figure 6(b) shows the relative change of pressure for fully elastic and elastic-plastic cases with different plastic indices. We observe that plastic contact and Gumbel distribution produce the closest result to Amonton's law.

Maugis [27] introduced two dimensionless parameters $\bar{P}=\frac{\mathrm{F}}{\pi R \Delta \gamma}$ and $=\frac{a}{\left(\frac{3 \pi \Delta \gamma R^{2}}{4 E^{*}}\right)^{1 / 3}}$ for force and area, respectively, and an adhesion parameter $\lambda=2 \sigma_{\text {adh }}\left(\frac{9 R}{16 \pi \Delta \gamma E^{* 2}}\right)^{1 / 3}$, where $\sigma_{\text {adh }}$ is the adhesion stress defined as:

$$
\sigma_{\text {adh }}=\frac{\Delta \gamma}{h_{0}}
$$



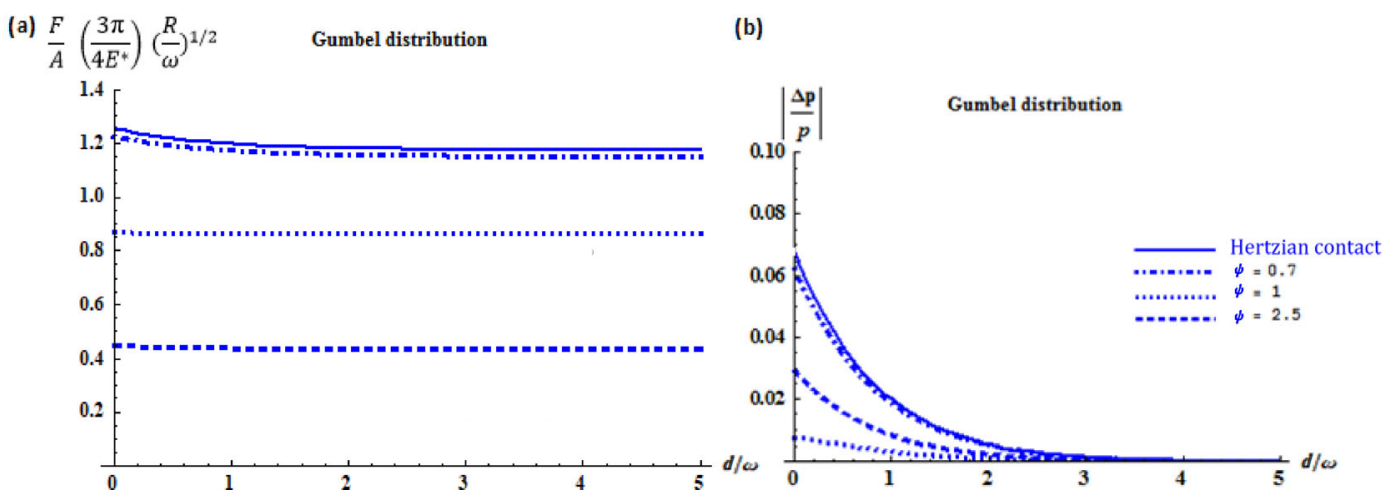

Fig. 6 (a) Pressure via separation and Elastic- plastic model as contact asperity. Variation of plasticity index changes the pressure since the real area of contact increases. (b) Relative change of pressure via separation. A fully elastic model such as Hertz model has the most pressure changes compared to elastic-plastic contacts.

where $\Delta \gamma$ is the surface energy and $z_{0}$ is the equilibrium in Lennard-Jones force, typically approximately $1 \AA$ [8].

$$
h_{0}=0.97 z_{0}
$$

If $\lambda>5$, the JKR analysis becomes appropriate and when $\lambda<0.1$, the DMT model is applicable. In the intermediate range $0.1<\lambda<5$, the MD model should be applied. For the adhesive contact problem in DMT limit, the pressure value is very close to the pressure in the Hertzian model. By increasing the adhesion parameter transition from DMT to JKR, a limit occurs and the pressure value decreases consequently (Fig. 7).

Considering adhesion with the MD model in contact, the results show that even asperities of heights $z<d$ can be in contact. Asperities with height $z>d$ are compressed and those with height $d-\delta_{\mathrm{c}}<z<d$ are stretched. $\delta_{\mathrm{c}}$ is the separation by which two surfaces become apart upon stretching them out. The effect of this pull-off force is considerable for a high value of $\lambda$ (Fig. 8).

Deviations from Amonton's law have been observed
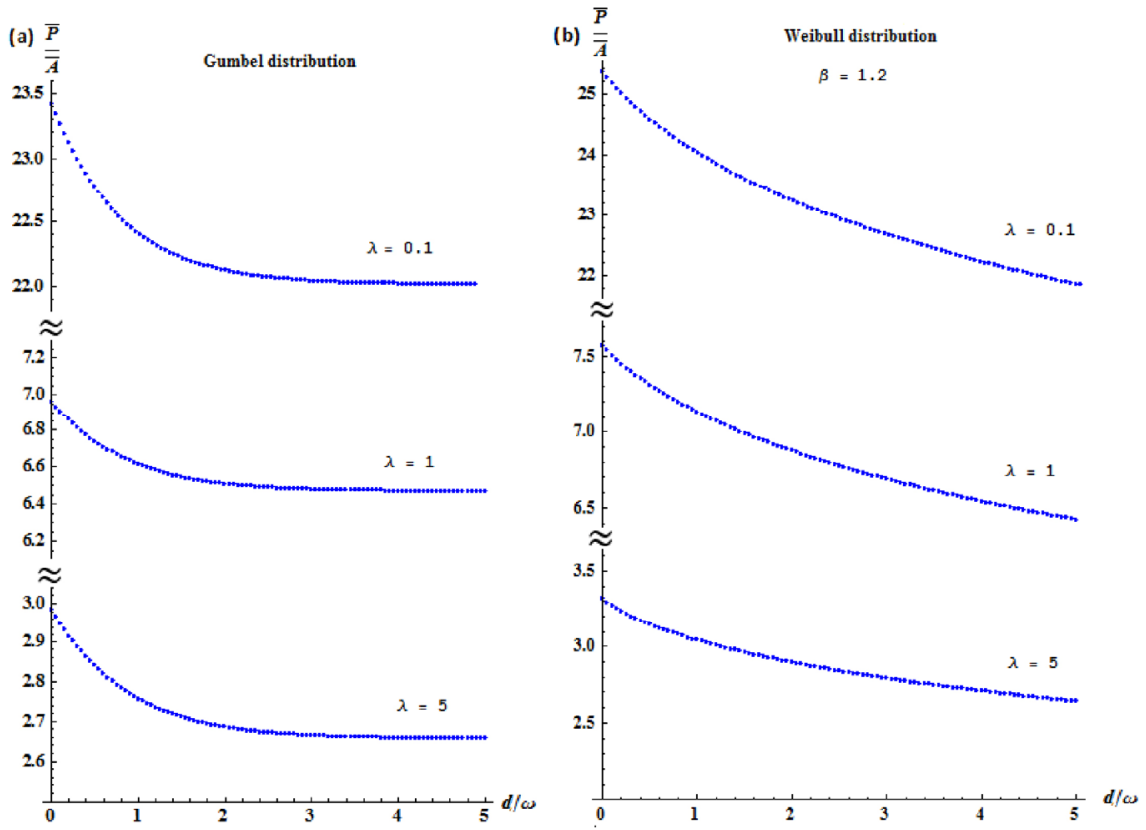

Fig. 7 (a) Gumbel distribution: pressure versus separation for contacts with different values of the adhesion parameter $\lambda$. For adhesive contacts, pressure has a lower value. (b) Weibull distribution $\beta=1.2$, pressure versus separation for contacts with different values of the adhesion parameter $\lambda$. 
by increasing the adhesion in materials [39]. Table 2 shows the results of Gumbel distribution for three values of the adhesion parameter and a fixed value of roughness, where we observe an increase in pressure with adhesion. We observe more deviation from Amonton's law for higher adhesion parameters. For a 10 times smoother surface with $\lambda=5$, the change in relative pressure is 0.22 , which is 10 times larger than that of a rougher surface.

Amonton-like behavior is dominant for a low adhesion parameter. Amonton's law does not describe friction behavior at zero or negative applied load. Adhesive control friction occurs for a higher value of adhesion parameter or a smoother surface. When

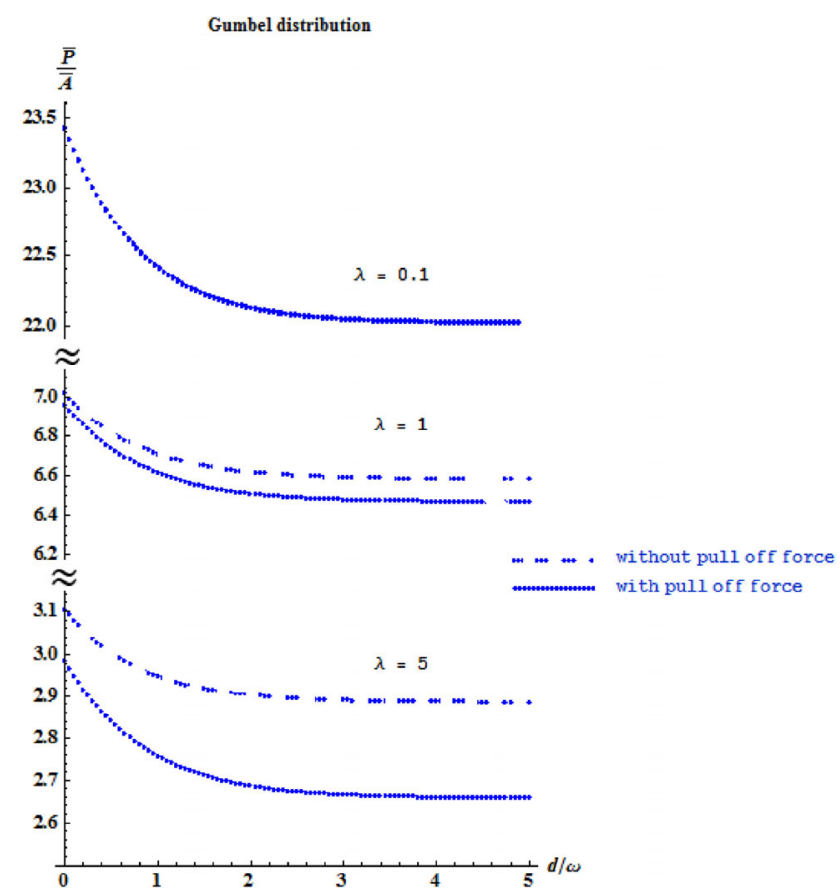

Fig. 8 In adhesive contacts, asperities with heights less than the separation of two surfaces are in contact. The pull off force is negligible for small adhesion parameters and it is more effective in high adhesion parameter.

Table 2 The pressure change in contact for Gumble distribution with different adhesion parameters. $p(d)=\frac{p(d)}{A(d)}$ is the dimesionless pressure in separation $d$, and $\omega$ is surface roughness.

\begin{tabular}{cc}
\hline$\lambda$ & $\frac{p(\omega)-p(5 \omega)}{p(5 \omega)}$ \\
\hline 0.1 & 0.013 \\
1 & 0.015 \\
5 & 0.022 \\
\hline
\end{tabular}

roughness decreases by 10 times for a fixed adhesion parameter, the pressure of contact falls significantly (Fig. 9).

\section{Beyond the GW model}

Whilst in GW model, an identical radius for all asperities is assumed, some authors have attempted to extend this to a more realistic setting by combining the BGT model with the GW model. In the BGT model, the parameter $\alpha$, which appears in Longuet-Higgins [22] and Nayak [23] probability distribution of summits for surface statistics of isotropic surface, is defined as $\alpha=\frac{m_{0} m_{4}}{m_{2}^{2}}$ where $m_{0}, m_{2}$, and $m_{4}$ are the zero, second, and fourth moments of the surface roughness power spectrum, respectively. The parameter $\alpha$ is an indication of the breadth of distribution of the radiuses of asperities.

For instance, Greenwood presented a simplified version of the BGT model in 2006 [40]. In this model, the summits are spheres with a distribution of the mean curvature $R_{\mathrm{G}}=\sqrt{R_{1} R_{2}}$. In another model presented in Ref. [41], the mean curvature of a summit is considered as $R_{\mathrm{A}}=\frac{2 R_{1} R_{2}}{R_{1}+R_{2}}$; we refer to it as the NT model. An improved model of the GW model is McCool [42], which combines the GW model and some results of the NT statistical model. We refer the reader to Ref. [43] for a detailed description of these models.

In order to test our proposal, we use Gumbel distribution as the summit distribution in the GWMcCool model. These results shows a better conformity with Amonton's law compared with those of other asperity models presented in Ref. [43]. In addition, the real area of contact has more realistic values compared with the models in Ref. [43] (Fig. 10). In Ref. [43], the heights and separation are scaled by the surface height variance $\sqrt{m_{0}}$ instead of the height variance of the summit. The relationship between $m_{0}$ and $\omega^{2}$ was determined by Bush et al. [44] as

$$
\omega^{2}=\left(1-\frac{0.8968}{\alpha}\right) m_{0}
$$



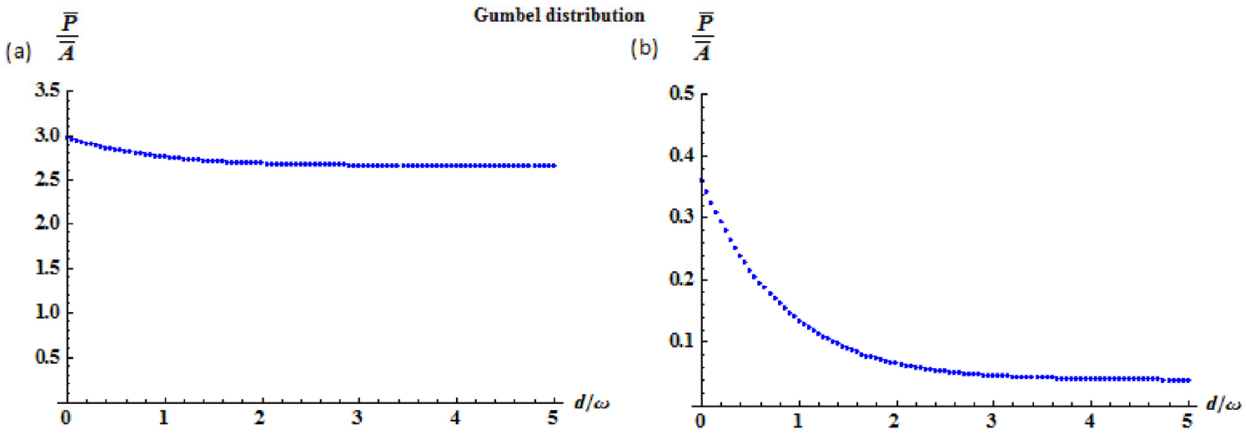

Fig. 9 Surface roughness decreases the adhesion effects in contact. (a) The pressure of contact for $\lambda=5$ in a fixed roughness. (b) The roughness of the surface decreased by 10 with $\lambda=5$ and resulted in considerable reduction in the pressure.
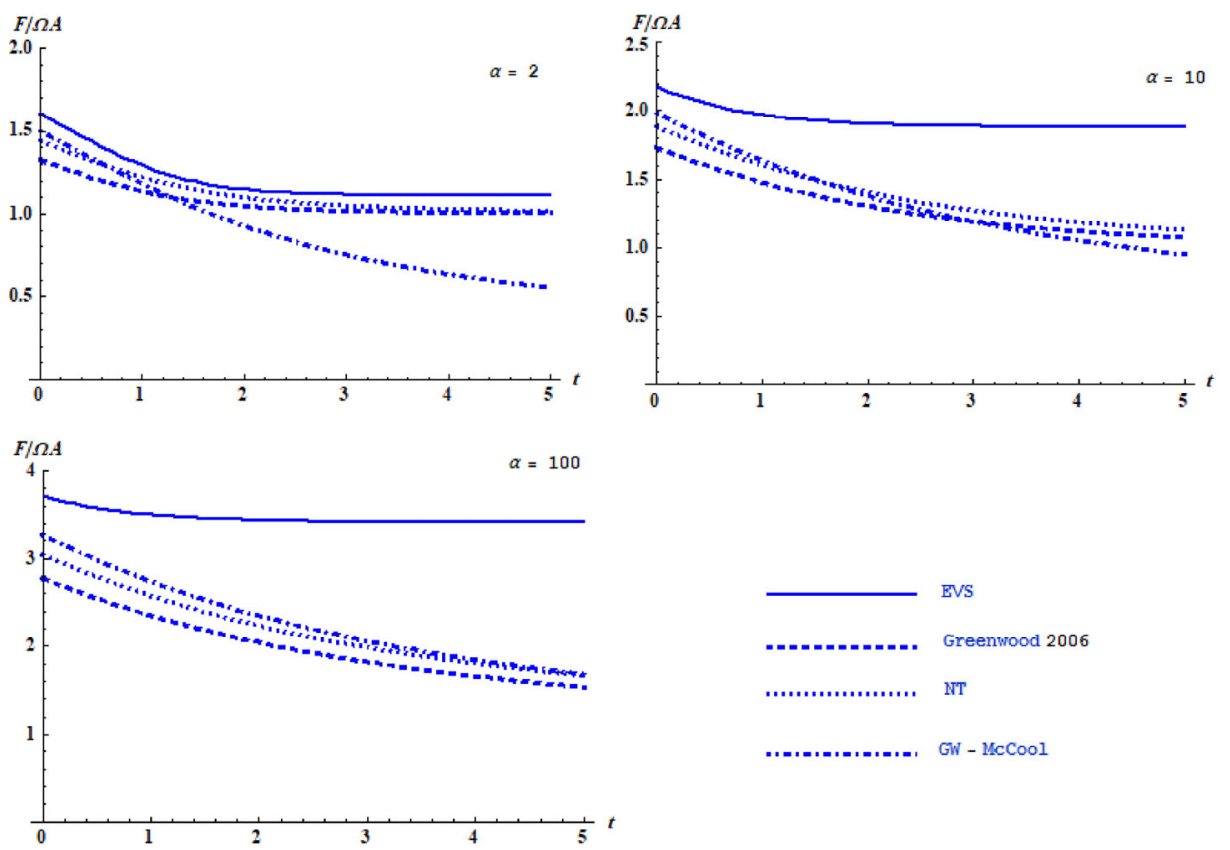

Fig. 10 When we use Gumbel distribution in GW- McCool model the value of pressure is more uniform than other models.

The summit variance approaches the surface variance as $\alpha$ becomes large. Table 3 shows the real area of contact when the separation is $\left(\sqrt{m_{0}}-5 \sqrt{m_{0}}\right)$ and Table 4 shows the pressure change at this distance. For a higher value of $\alpha$, Gumbel distribution has the most uniform pressure, which indicates the closest similarity with Amonton's law in a realistic area of contact.

Table 3 The real area of contact to the nominal area when the distance between two surfaces is $\left(\sqrt{m_{0}}-5 \sqrt{m_{0}}\right)$.

\begin{tabular}{ccccc}
\hline$\alpha$ & $\begin{array}{c}\text { Greenwood } \\
2006\end{array}$ & NT & GW-McCool & EVS \\
\hline 2 & $10^{-7}-0.061$ & $2 \times 10^{-8}-0.010$ & $3 \times 10^{-8}-0.062$ & $0.0001-0.085$ \\
10 & $10^{-7}-0.059$ & $1 \times 10^{-7}-0.051$ & $1 \times 10^{-7}-0.051$ & $0.0004-0.087$ \\
100 & $10^{-7}-0.101$ & $9 \times 10^{-8}-0.087$ & $9 \times 10^{-8}-0.079$ & $0.0010-0.167$ \\
\hline
\end{tabular}

Table 4 The pressure change in the distance $\left(\sqrt{m_{0}}-5 \sqrt{m_{0}}\right)$ for different models. More realistic surfaces have a high value of $\alpha$. We have the closest result to Amontons' law with Gumbel distribution in GW-McCool distribution.

\begin{tabular}{cccc}
\hline & $\alpha=2$ & $\alpha=10$ & $\alpha=100$ \\
\hline Greenwood 2006 & 0.129 & 0.373 & 0.537 \\
NT & 0.198 & 0.419 & 0.544 \\
GW-McCool & 1.124 & 0.722 & 0.630 \\
EVS & 0.164 & 0.047 & 0.026 \\
\hline
\end{tabular}

\section{Conclusions}

Amonton's law states that there is a linear relationship between the applied load and friction force. It is acceptable to consider a linear relationship between 
the friction force and the real area of contact. Therefore, the pressure of contact should remain constant.

In this work, we combined various models of singleasperity contact, including Hertzian, elastic-plastic, and adhesive contact models with EVS, to determine the summit distributions to verify Amonton's law.

In EVS theory, there are three kinds of universal distributions for IID variables, namely Fréchet, Gumbel, and Weibull distributions. The height of asperities in a real surface is strongly correlated. Here, we consider them as IID variables and use Fréchet, Gumbel, and Weibull distributions as the height of the summit. Surface roughness is a strongly correlated system and very little is known about EVS of correlated heights. For (1+1)-dimensional KPZ surface, the exact EVS distribution is Airy distribution. We extend Airy distribution to the two-dimensional surface and use it as the summit distribution. The resulting pressure varies considerably with the applied load.

Among EVS distributions, Gumbel distribution shows the best conformity with Amonton's law for Hertzian contact. One way to determine the relevance of the Gumbel distribution is to measure the height profile and determine the statistics of the height distribution. The pressure is almost constant for a relatively large interval of the applied load. Although Weibull distribution with $\beta=1$ is an exponential distribution and has a constant pressure with the applied load, it is not considered an appropriate candidate for summit distribution as the height distribution is uniform for $\beta=1$. Fréchet distributions with $\beta \leq 2$ are fat-tailed. They decay very slowly. For $\beta>2$, the number of short summits is not sufficient to reduce or balance the pressure at high loads and therefore, the pressure increases. We also combine Gumbel distribution with the GW-McCool model, which is an improved case of the GW model. Here, a bandwidth for wavelengths $\alpha$ is assumed. Comparison of this model with the original GW-McCool model and other simplified versions of BGT reveals that Gumbel distribution has a better conformity with Amonton's law for all values of $\alpha$.

The other point of interest is the best model for an asperity. Plastic deformations occur during contact. The changes in pressure are minimum with a combination of plastic and elastic deformations. When adhesion exists in contact, the main observation is that there is some friction force at zero or even negative applied load. Asperities with heights even less than the separation between the two surfaces are in contact. For a small value of adhesion parameter, Amonton-like behavior is dominant. The adhesion-controlled friction overcomes the load-controlled friction for a strong adhesion parameter. We also observed that adhesion increases for a surface with a lower value of roughness. We should extend this analysis to a more realistic case with the correlated height of asperity and use EVS of correlated variables and also consider the deformity of the asperities under pressure and changes in their geometry when the radius of curvature changes.

\section{Acknowledgement}

We are indebted to Daniel Bonn and Bart Weber for many detailed discussions on tribology.

Open Access: The articles published in this journal are distributed under the terms of the Creative Commons Attribution 4.0 International License (http:// creativecommons.org/licenses/by/4.0/), which permits unrestricted use, distribution, and reproduction in any medium, provided you give appropriate credit to the original author(s) and the source, provide a link to the Creative Commons license, and indicate if changes were made.

\section{References}

[1] Hurtado J A, Kim K S. Scale effects in friction of singleasperity contacts. I. From concurrent slip to singledislocation-assisted slip. Proc Roy Soc A: Math, Phys Eng Sci 455(1989): 3363-3384 (1999)

[2] Bhushan B, Nosonovsky M. Comprehensive model for scale effects in friction due to adhesion and two-and three-body deformation (plowing). Acta Mater 52(8): 2461-2474 (2004)

[3] Nosonovsky M, Bhushan B. Multiscale friction mechanisms and hierarchical surfaces in nano-and bio-tribology. Mater Sci Eng: R: Rep 58(3-5): 162-193 (2007)

[4] Adams G G, Müftü S, Azhar N M. A scale-dependent model for multi-asperity contact and friction. $J$ Tribol 125(4): 700-708 (2003)

[5] Bhushan B, Nosonovsky M. Scale effects in dry and wet friction, wear, and interface temperature. Nanotechnology 15(7): 749-761 (2004) 
[6] Popov V L. Contact Mechanics and Friction: Physical Principles and Applications. Berlin, Heidelberg (Germany): Springer, 2010.

[7] Coulomb C A. Theorie des Machines Simple (Theory of Simple Machines). Paris: Bachelier, 1821.

[8] Tolstoi D M. Significance of the normal degree of freedom and natural normal vibrations in contact friction. Wear 10(3): 199-213 (1967)

[9] Persson B N J. Sliding Friction: Physical Principles and Applications. Berlin Heidelberg (Germany): Springer, 2013.

[10] Gitis N V, Volpe L. Nature of static friction time dependence. J Phys D: Appl Phys 25(4): 605-612 (1992)

[11] Brockley C A, Davis H R. The time-dependence of static friction. J Lubr Technol 90(1): 35-41 (1968)

[12] Hosford W F. Solid Mechanics. Cambridge (UK): Cambridge University Press, 2010.

[13] Dieterich J H. Time-dependent friction in rocks. J Geophys Res 77(20): 3690-3697 (1972)

[14] Bowden F P, Tabor D. Friction and Lubrication of Solids, vol. I. Oxford (UK): Clarendon, 1950.

[15] Rabinowicz E. Friction and Wear of Materials. New York (USA): John Wiley \& Sons Inc., 1966.

[16] Tiwari A, Dorogin L, Steenwyk B, Warhadpande A, Motamedi M, Fortunato G, Ciaravola V, Persson B N J. Rubber friction directional asymmetry. EPL 116(6): 66002 (2017)

[17] He G, Müser M H, Robbins M O. Adsorbed layers and the origin of static friction. Science 284(5420): 1650-1652 (1999)

[18] Volmer A, Nattermann T. Towards a statistical theory of solid dry friction. Z Phys B Conden Matter 104(2): 363-371 (1997)

[19] Greenwood J A, Williamson J B P. Contact of nominally flat surfaces. Proc Roy Soc A: Math, Phys Eng Sci. 295(1442): 300-319 (1966)

[20] Archard J F. Elastic deformation and the laws of friction. Proc Roy Soc A: Math, Phys and Eng Sci 243(1233): 190-205 (1957)

[21] Bush A W, Gibson R D, Thomas T R. The elastic contact of a rough surface. Wear 35(1): 87-111 (1975)

[22] Longuet-Higgins M S. The statistical analysis of a random, moving surface. Philos Trans Roy Soc A: Math, Phys Eng Sci 249(966): 321-387 (1957)

[23] Nayak P R. Random process model of rough surfaces. $J$ Lubr Technol 93(3): 398-407 (1971)

[24] Persson B N J. Contact mechanics for randomly rough surfaces. Surf Sci Rep 61(4): 201-227 (2006)

[25] Coles S. An Introduction to Statistical Modeling of Extreme Values. Vol. 208. London (UK): Springer, 2001.

[26] Hertz H. On the contact of elastic solids. J Reine Angew
Math 92: 156-171 (1882)

[27] Maugis D. Adhesion of spheres: The JKR-DMT transition using a Dugdale model. J Colloid Interface Sci 150(1): 243-269 (1992)

[28] Chang W R, Etsion I, Bogy D B. An elastic-plastic model for the contact of rough surfaces. J Tribol 109(2): 257-263 (1987)

[29] Bury K. Statistical Distributions in Engineering. Cambridge (UK): Cambridge University Press, 1999.

[30] de Oliveira J T. Statistical Extremes and Applications. Vol. 131. Dordrecht (Netherlands): Springer, 1984.

[31] Al-Hasan M, Nigmatullin R R. Identification of the generalized Weibull distribution in wind speed data by the Eigencoordinates method. Renew Energy 28(1): 93-110 (2003)

[32] Huillet T, Raynaud H F. Rare events in a log-Weibull scenario-Application to earthquake magnitude data. Eur Phys J B-Conden Matter Complex Syst 12(3): 457-469 (1999)

[33] Gnedenko B. Sur la distribution limite du terme maximum d'une serie aleatoire. Ann Math 44(3): 423-453 (1943)

[34] Fisher R A, Tippett L H C. Limiting forms of the frequency distribution of the largest or smallest member of a sample. Math Proc Cambridge Philos Soc 24(2): 180-190 (1928)

[35] Einmahl H J, de Haan L. Empirical processes and statistics of extreme values, 1 and 2. AIO Course, available at www.few.eur.nl/few/people/ldehaan/aio/aiol.ps, www.few.eur.nl/ few/people/ldehaan/aio/aio2.ps.

[36] Majumdar S N, Comtet A. Exact maximal height distribution of fluctuating interfaces. Phys Rev Lett 92(22): 225501 (2004)

[37] Majumdar S N, Comtet A. Airy distribution function: From the area under a Brownian excursion to the maximal height of fluctuating interfaces. J Stat Phys 119(3-4): 777-826 (2005)

[38] Nuri K A, Halling J. The normal approach between rough flat surfaces in contact. Wear 32(1): 81-93 (1975)

[39] Pullen J, Williamson J B P. On the plastic contact of rough surfaces. Proc Roy Soc A: Math, Phys Eng Sci 327(1569): 159-173 (1972)

[40] Greenwood J A. A simplified elliptic model of rough surface contact. Wear 261(2): 191-200 (2006)

[41] Thomas T R. Rough surfaces. 2nd ed. Singapore: World Scientific, 1998.

[42] McCool J I. Comparison of models for the contact of rough surfaces. Wear 107(1): 37-60 (1986)

[43] Carbone G, Bottiglione F. Asperity contact theories: Do they predict linearity between contact area and load? $J$ Mech Phys Solids 56(8): 2555-2572 (2008)

[44] Bush A W, Gibson R D, Keogh G P. The limit of elastic deformation in the contact of rough surfaces. Mech Res Commun 3(3): 169-174 (1976) 


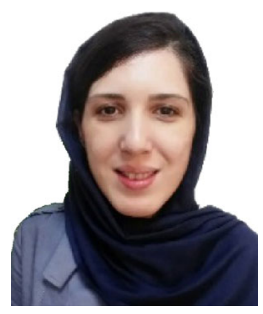

Azadeh MALEKAN. She received her B.S and M.S. degrees in physics from Imam Khomeini International University and University of Tehran,

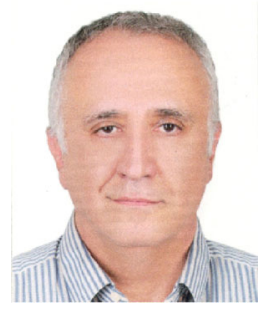

Shahin ROUHAI. He received his B.S and Ph.D. degrees in theoretical physics from University of Kent at Canterbury and Imperial College, London, UK, in 1977 and 1980, respectively. He is attached to the Sharif University of Technology as
Iran, in 2008 and 2011, respectively. She is a Ph.D. student at Sharif University of Technology from 2013 until now. Her research interests include critical phenomena and microtribology.

a professor of theoretical physics. He is a member of the research council of IPM and involved in the establishment and founding of IPM till present. His research interests include conformal field theory, critical phenomena, quantum field theory, and complex fluids. 